\title{
Methods and more
}

\author{
A brief look at the changes underway in Nature Methods as the journal continues to mature.
}

Last year marked the five year anniversary of Nature Methods. We are pleased with the community response to the journal: metrics and comments from our readers both show increasing impact of our content. Partially because of this success, we made only minor changes to the journal's offerings over this period, but the changes are now becoming more frequent as we strive to better serve our readers.

In 2008, we discontinued the Protocol article type after Nature Protocols launched as a dedicated venue for step-by-step descriptions of previously published methods. That same year, we began offering two new venues for presenting original research that we believe will prove valuable for many scientific communities: Analyses and Resources. Analysis articles contain thorough and objective performance comparisons of existing methods and tools. Resource papers describe new large collections of tools or data.

Original research, reviews and commentaries represent the majority of content in Nature Methods, and we are indebted to the scientists who serve as our authors and reviewers. Accompanying this extensive content, the Research Highlights in Nature Methods allow our editors to communicate high-interest methodological developments published in other journals. By describing the work in a short, engaging format we hope our readers will discover interesting methods they might otherwise overlook and possibly insights that might not be gleaned from the original manuscript. This year we began including a greater number of these highlights in each issue.

As of January of this year we also chose to focus on Nature Methods authors in a new piece called 'The Author File. This monthly column provides a behindthe-scenes view of the work that went into a research paper appearing in that issue of the journal and of the primary author or authors responsible for it. It also includes their outlook on how methods could be used in other laboratories.

These sections are part of an effort to provide our readers with more general interest journalistic content relevant to practicing researchers. In this issue, we are happy to announce a new monthly column on visual presentation of scientific data. This past April we published a well-received Focus on visualizing biological data that covered the use of visualization techniques and software. The new column, 'Points of View', deals with fundamental aspects of visual presentation applicable to anyone who works with visual representations of data. Content in this column will be more along the lines of the information in the classic text The Visual Display of Quantitative Information by Edward Tufte ( $2^{\text {nd }}$ edn.; Graphics Press, 2001) than the specialized information in the Focus.

The column author, Bang Wong, is the creative director of the Broad Institute in Cambridge, Massachusetts, USA where he works at the interface of science and art. He led the effort to design and build the Broad Institute's small museum, the DNAtrium, and established a data visualization initiative to develop methods for visually exploring large data sets. In addition to these efforts, he provides hands-on assistance to researchers on the communication of their experimental results to the scientific community through graphics and data visualization.

At Nature Methods we witness authors' struggles to display data clearly and with minimal bias. Everything from choice of colors, fonts, graph type and design to overall layout can influence data interpretation. Poor choices can have a range of effects. Figures or presentations may be unattractive but otherwise serve their purpose. Alternatively, figures may be difficult to interpret—or more worryingly—misleading. Each month Wong will focus on a particular aspect of data presentation or visualization and provide easy-to-apply tips on how to overcome common pitfalls. This month's column is on the use of color in scientific figures, a commonly mishandled aspect of figure design.

Admittedly, some journals' style rules may preclude researchers from following all advice. We expect that we may learn a thing or two ourselves. But many of the tips will be easily implementable, and published papers are not the only place where researchers present their data. PowerPoint presentations and posters often suffer the most from poor presentation. Interestingly, Tufte contends that distributing a brief written report-readable in five to ten minutes-followed by open discussion, is a superior method of transferring knowledge. But PowerPoint and poster presentations will probably be with us for the foreseeable future, and tips to make the information presented in them easier to grasp in less time would clearly benefit the scientific community.

We hope our readers find these additions to Nature Methods useful, and we urge you to let us know what you think, including suggestions of additions you would like to see. Your feedback will help us in our goal of providing valuable and engaging content. 